SERgio De Zubiría*

\title{
EDUCACIÓN, JUSTICIA SOCIAL Y DIVERSIDAD CULTURAL
}

Colón ha descubierto América, pero no a los americanos. Toda la historia del descubrimiento de América, primer episodio de la conquista, lleva la marca de esta ambigüedad: la alteridad humana se revela y se niega a la vez.

Tzvetan Todorov

\section{Resumen}

Esta reflexión intenta ser un aporte en la discusión conceptual sobre las relaciones entre cultura-educación, una reflexión sobre sus incidencias en la justicia social y una contribución para la orientación en la formulación de políticas educativas atentas a la diversidad cultural. Constituye la reiteración constante de los grandes desafíos que contiene la dimensión cultural para construir una educación respetuosa de la diferencia. No pretende ser una fundamentación completa, sino un material en construcción; su intención es indicar unos puntos de atención en este difícil recorrido por los retos actuales de la educación. Se abordan tres grandes temas: 1) el reconocimiento institucional en el campo educativo de la importancia de la diversidad, a partir de la década de los noventa del siglo XX; 2) unas breves aproximaciones a la importancia del reconocimiento de la diversidad cultural para abordar los problemas de la justicia contemporánea, y 3) algunas propuestas de tipologías para comprender una educación que enfrente el reto del reconocimiento de la diversidad cultural.

Palabras clave: Educación, cultura, justicia social, diversidad cultural, políticas educativas.

\section{EDUCATION, SOCIAL FAIRNESS AND CULTURAL DIVERSITY}

\section{Abstract}

This reflection aims at being a contribution on the conceptual discussion among culture-education. A reflection of its consequences on social fairness, and a contribution to address the formulation of educational politics, in view of cultural diversity. It establishes the constant repetition of significant challenges inside cultural dimension to construct a respectful education on human diversity. It doesn't attempt to be a complete basis, but an action in process. Its objective is presenting some remarkable points on the hard route through actual challenges on educational field. Three important topics are tackled: 1) Institutional acknowledgment on the educational field of diversity significance, arising from nineties decade in century $x x$. 2) some briefly approximations to the importance of cultural diversity to undertake the problems of contemporary fairness; and 3) some proposals to understand an education which faces the acknowledgment of cultural diversity.

Key words: Education, culture, social fairness, cultural diversity, educational politics.

* Doctor en Filosofía Política. Máster Internacional en Gestión, políticas culturales y desarrollo, Universidad de Girona y Unesco. Profesor del Departamento de Filosofía de la Universidad de los Andes, Bogotá. sde@uniandes.edu.co

Texto original recibido: 22-04-06 y aprobado: 18-05-06 


\section{LA DIVERSIDAD CULTURAL EN EL DISCURSO EDUCATIVO}

A partir de los primeros años de la década de los noventa del siglo $X X$, el grupo de intelectuales e investigadores que conformaron el Club de Roma iniciaron los primeros diagnósticos sobre la problemática global mundial que caracterizaría los días venideros, intentando análisis prospectivos de larga duración. Además de ciertos "viejos" problemas que tenderían a agudizarse -tales como las disparidades económicas cada vez mayores entre el norte y el sur, el crecimiento del número de seres humanos viviendo bajo la línea de pobreza crítica, las migraciones masivas hacia países con mayor bienestar material y la degradación general del medio ambiente-, las próximas décadas traerían problemas aún no conocidos en la historia occidental, en relación con la virulencia del racismo, la xenofobia, el hipernacionalismo, los fundamentalismos religiosos, la intolerancia, la aporofobia, la violencia y las violaciones generalizadas de los derechos humanos. En este sentido, el mundo que empezaba a aparecer ante nuestros ojos se distancia mucho de reproducir la apariencia de unidad y homogeneidad características de la guerra fría. Y la pretendida cohesión política y social empezaría a ceder ante conflictos que considerábamos ya terminados: las luchas interétnicas y religiosas, la discriminación ciudadana y sexual, la intolerancia con la diversidad y profundas fracturas en el tejido profundo de las sociedades actuales.

Frente a este panorama sombrío, esas fracturas vendrían a tomar el lugar metafórico de la explosión atómica que no se produjo, y los desafíos tendrían que provenir de dos escenarios centrales: el análisis de las cinco revoluciones que están en curso, y de una profunda reflexión sobre las relaciones entre educación, cultura y desarrollo. Es por ello que la Unesco en los años noventa decide convocar una "comisión internacional sobre cultura y desarrollo" que intentara enfrentar el incierto desafío de estos dos complejos escenarios.

El informe de la Comisión Mundial de Cultura y Desarrollo, publicado en 1997, plantea la necesidad de repensar las relaciones entre cultura, educación y desarrollo en puntos centrales tales como: la necesidad de un enfoque endógeno del desarrollo que tenga siempre en cuenta el contexto sociocultural, en el cual el

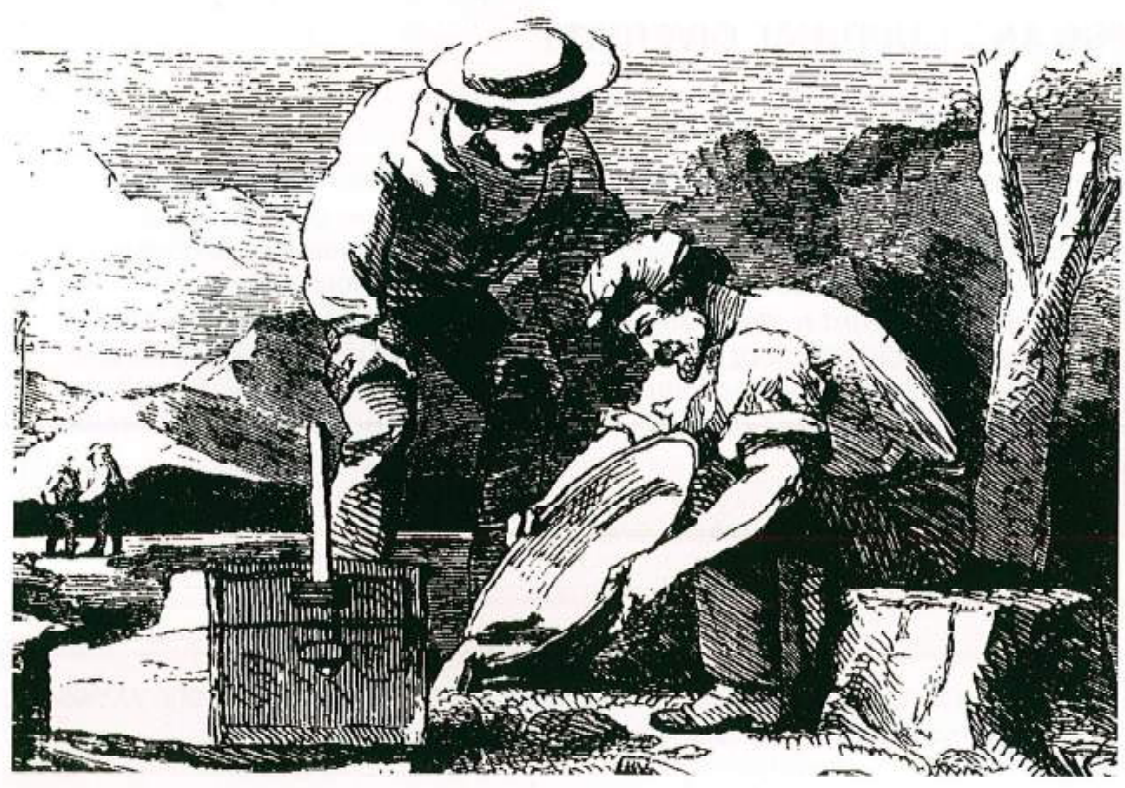

desarrollo debe realizarse, así como las condiciones específicas vinculadas a una determinada cultura; este enfoque asigna a la población beneficiaria una función activa en su propio desarrollo que integre siempre sus propias estructuras socioculturales y, por tanto, sólo es posible con la activa participación de las comunidades interesadas. Muchos de los obstáculos y las dificultades del desarrollo se deben al irrespeto del carácter endógeno y a las condiciones socioculturales de las comunidades. Otro punto central sería la idea de una ecuación del desarrollo que puede integrar factores más allá de los económicos, por ejemplo la elección autónoma del tipo de desarrollo, la deliberación propia de las tecnologías que hay que importar, adaptar o crear, la decisión de las formas de organización y significado de los valores culturales y la construcción creativa del contenido del sistema educativo. Si la cultura es entonces aquella llave que abre las puertas de potencial creador de una comunidad o de una sociedad, la educación es la dimensión que puede configurar y guiar el desarrollo de ese potencial. En términos de la Comisión, tiene que existir una interacción constante entre cultura y educación, porque si la primera es la que ilumina, la segunda da la necesaria perspectiva.

Es en el informe a la Unesco de la Comisión Internacional sobre la Educación para el siglo XXI, coordinada por Jacques Delors (1996), donde se plasman todas estas preocupaciones, horizontes y tensiones en el campo de la educación. En tres órdenes se constatan profundos desencantos: ante el desarrollo económico y social, la posible reducción de la guerra, y la posibilidad de aprender a vivir juntos. Experimentamos grandes desilusiones en el posible progreso económico y social que se manifiestan en el aumento del desempleo y de los fenómenos de exclusión, como también en el mantenimiento de las desigualdades de desarrollo en el mundo actual. Quienes vieron en el final de la guerra fría la perspectiva de un mundo más pacífico, viven 
el desencanto del incremento de la violencia y las guerras, hasta tal punto que después de la caída del muro de Berlín se han presentado muchísimas guerras entre naciones, entre grupos étnicos, en relación con injusticias acumuladas en los planos económico y social, contra la droga y el terrorismo y muchos enemigos imaginarios. Las dificultades que enfrenta el aprender a vivir juntos en una posible "aldea planetaria", si no podemos ni siquiera vivir en esas comunidades a las que pertenecemos casi por naturaleza: la nación, la región, la ciudad, el barrio, el pueblo, la vecindad, la escuela, etc... Las principales tensiones que, sin ser nuevas, están en el centro de la problemática en el siglo XXI, como expresión también de esos desencantos, son por lo menos seis.

En primer lugar, la tensión entre lo mundial y lo local como manifestación de la construcción de un ciudadano que, respetuoso de lo mundial, no pierda sus raíces y participe activamente en la vida de su nación y de sus comunidades de base. De un tipo de ciudadano que, sin renunciar a su vocación de escoger su destino y realizar sus potencialidades, no abandone su preocupación por los proyectos colectivos de una vida en común. En segunda instancia, la tensión entre tradición y modernidad como el interrogante del tipo de modernidad que deseamos y si ésta necesariamente va a destruir nuestras propias tradiciones; o la posibilidad de un tipo de modernidad que también respete y potencie las tradiciones culturales. En tercer lugar, ante las nuevas experiencias del tiempo como imperio de lo efímero y la instantaneidad, las tensiones entre el largo y el corto plazo en todos los ámbitos de la vida humana. Entre unas políticas educativas que logren sensibilizarse tanto a los proyectos de largo plazo como a las transformaciones de corto plazo. En cuarto lugar, la cuestión clásica de la justicia educativa entre la equidad como igualdad de oportunidades y lo relativo a la competencia, en un mundo donde cada vez se habla y discute más sobre lo relativo a los límites y las posibilidades de la competencia. En quinta instancia, la tensión entre el extraordinario y rápido desarrollo de los conocimientos y las capacidades de asimilación del ser humano; entre el carácter perenne y el carácter efímero que traen como consecuencia las actuales revoluciones científicas y tecnológicas. Y por último, la tensión entre la mercantilización de la vida y los valores humanos, y la importancia de los principios y los valores que luchan contra la mercantilización. La revalorización de los aspectos éticos y culturales de la educación o el primado exclusivo de la ganancia y el ascenso social.

En este panorama la respuesta educativa tendría que estar basada en cuatro pilares o fundamentos: aprender a conocer, aprender a hacer, aprender a vivir juntos en medio de la diversidad, y aprender a ser. Una educación que no se estructure a partir de estos cuatro ejes no podrá resolver las tensiones y los desafíos de la educación para el siglo XXI.

Aprender a conocer, no en el sentido de la adquisición de conocimientos clasificados y codificados previamente, sino aprender a comprender el mundo que nos rodea, en forma suficiente para vivir con dignidad, desarrollar nuestras potencialidades y comunicarnos con los demás, cuyo fin no es una función operativa sino el placer que contiene el comprender, el conocer y el descubrir. Renunciando a la idea de un conocer enciclopédico como también de carácter omnisciente. Un aprender a conocer que nos aboque a una cultura integral, a la apertura a otros lenguajes y conocimientos y a la posibilidad de la comunicación. Aprender a hacer, no en el simple significado de preparar a alguien para una tarea material predefinida o la fabricación de algo, tampoco la mera transmisión de prácticas rutinarias, sino a apropiar caminos y procesos que posibiliten innovaciones en la acción del hacer. Que las tareas puramente físicas sean suplantadas por actividades mucho más intelectuales y concep- tuales. Aprender a vivir juntos dentro del diálogo y respeto a la diversidad es, sin duda, una de las más difíciles empresas de la educación contemporánea. No basta simplemente enseñar la no violencia o el repudio a cualquier forma de discriminación, sino buscar orientaciones adecuadas para el descubrimiento gradual del otro en su diferencia y el incremento en la participación en proyectos comunes en medio de la diversidad. La educación tendría que buscar métodos adecuados para solucionar una doble misión: enseñar a reconocer las diversidades de todo tipo y contribuir a fomentar la interdependencia entre seres humanos diversos. Un descubrimiento del otro distinto a mí, que también pasa forzosamente por el reconocimiento de uno mismo. Aprender a ser, fue el título del informe sobre educación de la Unesco de 1972, el cual manifestaba en su preámbulo el temor a una deshumanización del mundo vinculada a las consecuencias de una evolución exclusivamente tecnológica.

La Agenda del siglo XXI para la educación en América Latina y el Caribe (1998), promovida por la Organización de Naciones Unidas, sostiene que las políticas educativas en la región enfrentan un conjunto de tensiones y decisiones centrales para orientar la educación hacia el siglo XXI. Las que se destacan son las siguientes: a) privilegiar los asuntos sociales de la educación o los problemas académicos; b) delegar en otros agentes la evaluación y el control de la educación o mantener transformada la regulación estatal; c) una descentralización para mejorar el servicio educativo o una descentralización para que los gobiernos nacionales se "desembaracen" del servicio educativo; d) privilegiar los aspectos normativos legales de las reformas educativas o convencer y persuadir a la ciudadanía; e) optar por el papel y la función del maestro o apostarle a otros apoyos y ayudas educativas. Pero es en el prólogo de esa agenda del siglo XXI, elaborado por Carlos Fuentes, donde se insiste con más vehemencia sobre la importancia de 
la diversidad cultural y sus tensiones con el orden económico y político. Para este escritor, los dramas de América Latina en vísperas del siglo XXI son profundos y desafiantes: una continuidad cultural que no ha encontrado aún una continuidad económica y política comparable; no hemos logrado la identificación entre la nación y las culturas; le hemos dado preferencia a la ideología, celebramos a la cultura en sus aniversarios y en la plaza pública, pero no le damos entrada en nuestros salones; algo está podrido en los reinos latinos de América cuando la mitad de la población sobrevive apenas con ingresos de menos de noventa dólares al mes; nuestras diferencias nos siguen separando forzadamente y no hemos podido hacer de esas diferencias virtudes; la sabiduría clásica nos dice que de la diversidad nace la verdadera unidad y la actual experiencia contemporánea nos recuerda que el respeto a las diferencias crea las fortalezas, y su negación, la debilidad. Por eso para este ensayista sólo será posible una educación latinoamericana que atienda a las particularidades nacionales y regionales del continente, que nos devuelva la confianza en que es desde nuestra diversidad respetada que nacerá una unidad respetable; unidos porque nos enriquecen nuestras diferencias.

\section{LOS PROBLEMAS DEL RECONOCIMIENTO EN LA JUSTICIA SOCIAL}

Las reivindicaciones de justicia social en el mundo actual pueden diferenciarse en dos tipos cada vez más claros: el primero, y también el más reconocido, hace referencia a la redistribución que postula una distribución más justa de bienes, servicios y recursos; y el segundo tipo, la exigencia de justicia en relación con las políticas del reconocimiento, que implica la asunción de diferencias culturales, étnicas, raciales, sexuales y de género, sin que se imponga una homogeneidad dominante y se pueda potencializar el reconocimiento

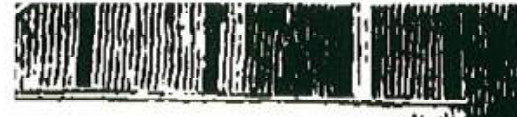

NEROSDIII CIN HI Hill RICH C NAP, Ol, Q Q

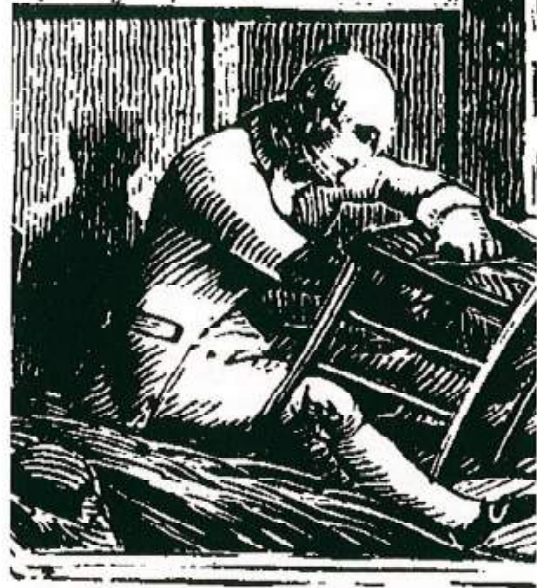

de estas diversidades. Las primeras han sido denominadas en el campo de la justicia social como "políticas de la redistribución"; las segundas, han sido apeladas "políticas del reconocimiento y la identidad". Por ejemplo, para la filósofa política Nancy Fraser, el objetivo que resulta más viable en justicia social, es un mundo que acepte las diferencias de un modo amistoso, en donde el asimilar las normas culturales dominantes o de la mayoría ya no tenga el precio de un respeto homogéneo de carácter abstracto.

La emergencia de estos dos tipos de reivindicaciones de justicia social ha configurado tanto una nueva constelación en el ámbito de la justicia, como nuevas problemáticas que necesariamente hay que enfrentar. En esta nueva constelación se pueden presentar alternativas divergentes como las siguientes: las reivindicaciones relevantes siguen siendo la distribución de los bienes y la riqueza material; hay que abordar en las condiciones actuales la distinción entre reivindicaciones de distribución y reivindicaciones de reconocimiento; deben predominar las políticas del reconocimiento sobre las políticas de redistribución. A la primera alternativa la podríamos identificar como la ceguera frente a la especificidad del problema del reconocimiento; a la segunda posición, como una especie de dicotomía o dualismo entre distribución y reconocimiento; y a la tercera, como el predominio exclusivo del reconocimiento o el auge de ciertas "políticas de identidad".

Desde el diagnóstico realizado por Nancy Fraser, los dos tipos de reivindicaciones de justicia hoy tienden a disociarse tanto en lo práctico como en lo intelectual. En la academia estadounidense se muestra, por ejemplo, la insistente tendencia a desvincular la política cultural de la diferencia y la política social de la igualdad (ejemplos ilustrativos son las posiciones de Richard Rorty, quien insiste en que la política de identidad es una desviación contraproducente para los temas económicos reales porque "balcaniza" a los grupos sociales y conlleva el rechazo a normas universales que constituyen las bases de la igualdad social; o el caso de Charles Taylor, quien insiste en que algunas políticas de redistribución lo que hacen es reforzar la injusticia). Dentro de los movimientos sociales las tendencias que reclaman la redistribución como remedio a la injusticia social se separan cada vez más de las tendencias que giran en torno al reconocimiento. Experimentamos, al mismo tiempo, una especie de polarización y también de unilateralidad, que a través de antítesis teóricas falsas se convierten en lugares comunes tales como: o redistribución o reconocimiento, política de clase o política de identidad, igualdad o diferencia, pluralismo o igualdad social.

La tesis fuerte que sostiene esta investigadora es que la justicia en las sociedades actuales "requiere tanto de la redistribución como del reconocimiento, ya que ninguna de las dos vías, por sí sola, es suficiente". Para ello, es necesario construir un concepto bivalente o bifronte de la justicia que cumpla al mismo tiempo dos condiciones: lograr reconciliar 
las reivindicaciones que defienden la igualdad social con las que defienden el reconocimiento de la diferencia, y plantear prácticamente cómo se da esa combinación. Una teoría bivalente o bifronte de la justicia implica integrar la redistribución y el reconocimiento dentro de un único marco conceptual que pueda superar las disociaciones políticas, divulgadas actualmente.

La construcción de este único marco conceptual supone abordar tres clases de problemas: los relativos al ámbito normativo filosófico, que conciernen a la relación entre la justicia del reconocimiento y la de la distribución; las cuestiones teórico-sociales, que atañen a la relación entre la economía y la cultura; y los asuntos políticos, programáticos, que se refieren a las tensiones prácticas que surgen de los esfuerzos políticos para promover simultáneamente redistribución y reconocimiento. Por tratarse este acápite de la naturaleza o carácter de la justicia social, aludiremos exclusivamente a las cuestiones de carácter normativo-filosóficas, que en el fundamento se pueden resumir en tres grandes interrogantes: ¿Es el reconocimiento un asunto que concierne a la justicia o un asunto de autorrealización? ¿Constituyen la justicia distributiva y la justicia de reconocimiento dos paradigmas distintos, o puede ser uno subsumido por el otro? ¿Requiere la justicia un reconocimiento de las diferencias de individuos o grupos, 0 será suficiente el reconocimiento de nuestra condición humana común?

Las posibles respuestas a estos profundos interrogantes son las cuestiones filosófico-normativas fundamentales. El reconocimiento de la diversidad cultural es un problema típico de justicia social y no una cuestión de autorrealización personal. Es injusto que a unos individuos o grupos les esté negada la condición de participantes plenos en la interacción social, por menosprecio a sus características diferenciales o a las características diferenciales atribuidas a ellos. La falta de reconocimiento no es simplemente una cuestión de actitudes perjudiciales que llevan a daños psicológicos, sino un problema radical de las estructuras socialmente establecidas en el ámbito de la equidad, la interpretación y la valoración. Presupone, normativamente, que toda persona tiene el mismo derecho a perseguir la estimación social en unas condiciones justas de igualdad de oportunidades. "Los modelos culturales dominantes de interpretación degradan fuertemente la feminidad, el no ser blanco, la homosexualidad y todo lo culturalmente ligado a ello. Cuando se da este caso, mujeres o personas de color o gays y lesbianas. se enfrentan con obstáculos, que no encuentran otros, en la búsqueda de estimación".

En cuanto a si se puede reducir la justicia distributiva a la de reconocimiento o viceversa, la propuesta del concepto bivalente de justicia implica la "innecesariedad" filosófica de reducir el uno al otro. Para justificar esta irreductibilidad, acuña nuestra pensadora la noción de "paridad participativa"; esta noción implica un postulado teórico y dos precondiciones adicionales. El postulado teórico sostiene que la paridad participativa es posible si se establecen normas básicas de igualdad legal formal, que son condiciones necesarias, pero no suficientes. "La justicia requiere acuerdos sociales que les permitan a todos los miembros (adultos) de la sociedad interactuar unos con otros como iguales". La primera precondición adicional es que la distribución de recursos materiales debe ser de tal manera que asegure la independencia y la voz de los participantes; y la segunda precondición adicional exige que los modelos culturales de interpretación y valoración sean de tal manera, que permitan expresar un respeto mutuo para todos los participantes y aseguren la igualdad de oportunidades para conseguir la estimación social. Al asegurar la paridad participativa, tanto la redistribución como el reconocimiento, se puede entonces hablar de un concepto bivalente de justicia y de lo innecesario de reducir el uno al otro.

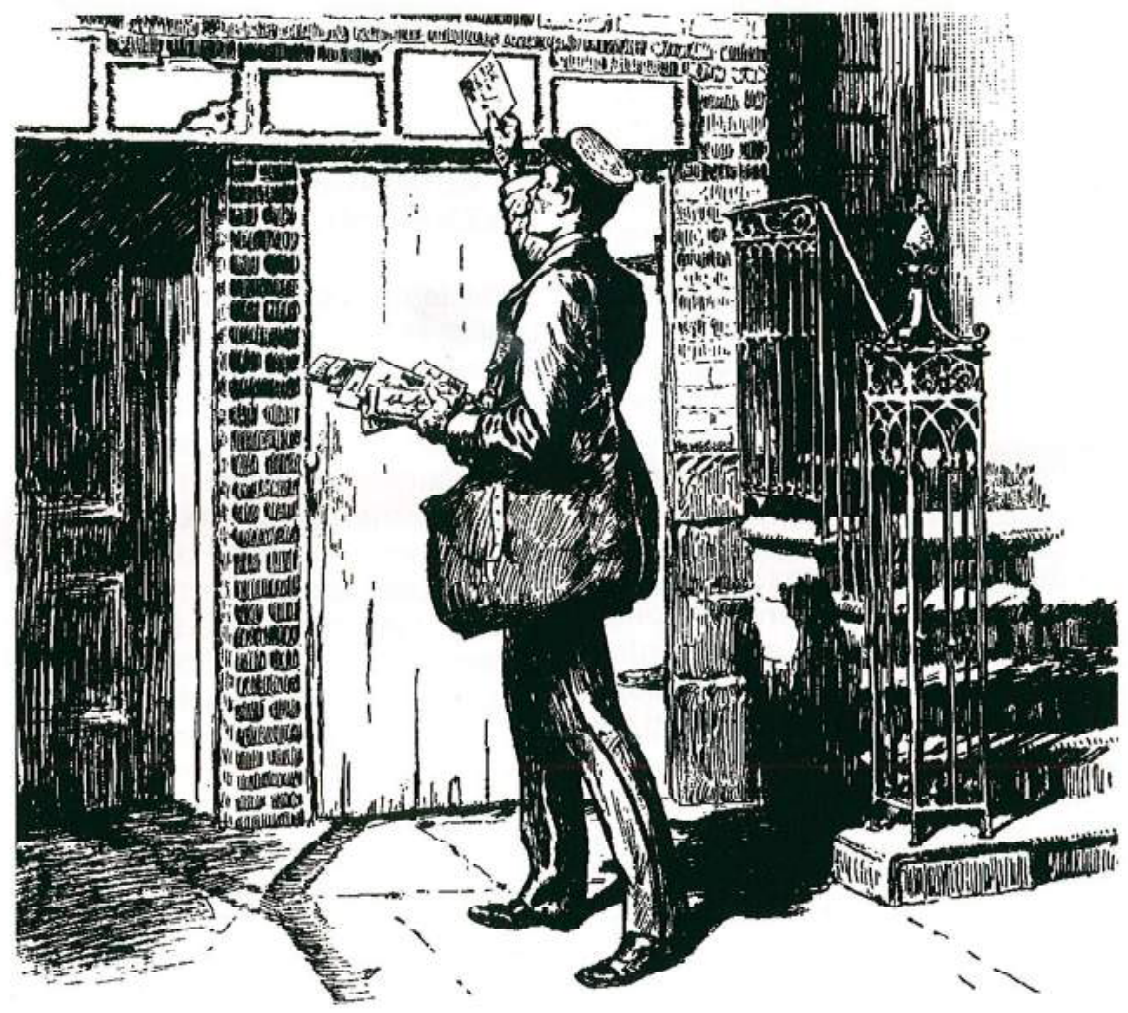


En cuanto a la justicia del reconocimiento de las diferencias de individuos o grupos, por encima o más allá del reconocimiento de nuestra condición humana común, la posición de Nancy Fraser es resaltar que la paridad participativa es una norma universalista en dos sentidos: por un lado, incluye a todos los participantes en la interacción y, por otro lado, presupone un valor moral de igualdad para todos los seres humanos. Pero esta posición, reconoce ella, podría ser objetada por cierto universalismo abstracto; entonces recurre para salir de esta objeción a una teoría social pragmática y crítica al mismo tiempo.

Todo depende precisamente de lo que la gente que no es reconocida necesita para poder participar en condiciones de igualdad en la vida social. Y no existe razón para asumir que todos precisen de lo mismo en todos los contextos. En algunos casos puede que se necesite que se quiten las diferencias excesivamente adscritas o construidas para poder participar como miembros con pleno derecho en la interacción. En otros casos puede que se deba tener en cuenta la diferencia insuficientemente reconocida. Aún en otros casos más, quizá se necesite enfocar a grupos dominantes o aventajados, destacando que la diferencia de éstos ha sido falsamente proclamada como universalidad. Alternativamente, puede que se necesite de-construir los términos mismos en los que las diferencias atribuidas están actualmente elaboradas. Finalmente, es posible que se necesite todo lo ya mencionado, o varios aspectos de ello, en combinación uno con otro y en combinación con la redistribución.

Es decir, en cuestiones del reconocimiento de las diferencias nunca son suficientes los argumentos filosóficos abstractos; se necesita ineludiblemente una teoría social crítica, orientada normativamente, muy informada empíricamente y guiada por el ideal de superar la injusticia, la cual debe plasmarse prácticamente en modelos concretos de educación y formación social.
Además de no ser nuevas las cuestiones sobre educación y justicia, como agudamente lo evidencia aquel texto fundacional de la pedagogía, la República de Platón, existe relativa conciencia de que el papel de la educación en asuntos de justicia social será cada vez mayor en las circunstancias actuales. Según el trabajo del investigador educativo R. W. Connell sobre escuelas y justicia social, existen tres razones fundamentales que avalan sus ineludibles relaciones: el sistema educativo es un bien público de gran importancia; parece evidente que esa importancia se incrementará en el futuro; si el sistema educativo trata injustamente a algunos de sus alumnos, no son éstos los únicos que padecen, sino que la calidad de la educación de todos los demás se degrada.

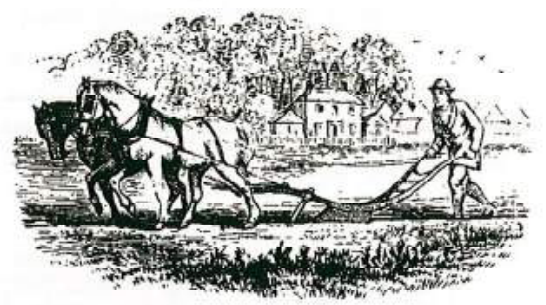

\section{POSIBLES TIPOLOGÍAS DE EDUCACIÓN EN LA DIVERSIDAD CULTURAL}

Toda forma de educación histórica implica mecanismos y procedimientos para diferenciar personas, épocas, situaciones, acciones, objetos, culturas, etc. Implícita, latente o explícitamente, consciente o inconscientemente, en el acto educativo clasificamos, ordenamos, priorizamos, dignificamos, alejamos, acercamos, invisibilizamos, etc. Por tanto, la diferencia y aquellos procesos por los que la construimos son de importancia fundamental en el reconocimiento de la diversidad. Las formas de asumir esa diversidad marcan una idea concreta de sociedad y de justicia social. En la educación multicultural esa importancia se deriva de tres preguntas o problemáticas centrales: 1) Los procesos de diferenciación y construcción de diferencia que realizamos "inevitablemente" en la acción educativa, ¿contienen o no injusticia, exclusión, discriminación o desigualdad?; 2) ¿Qué tipos de educación promueven la desigualdad a través de las diferencias y cuáles procesos educativos no lo hacen?; 3 ) ¿Son suficientes las oportunidades educativas que aludan a la redistribución de bienes y servicios, o también son necesarias las políticas del reconocimiento de la diversidad y las identidades?

Las posiciones ante estos interrogantes, para Peter McLaren, marcarán tres posturas filosóficas distintas ante el fenómeno del multiculturalismo: conservador, liberal y crítico. El multiculturalismo "conservador" persiste en la necesidad de un mundo con una "cultura en común" que porta los "verdaderos" valores de la "civilización" humana; el enfoque "liberal" defiende las tesis de un "pluralismo tolerante", y considera que las diferencias de sexo, raza, clase, no son centrales en la idea de "ciudadanía" porque lo determinante es la igualdad "formal" ante la ley; la perspectiva del multiculturalismo "crítico" concibe las representaciones diferenciales de clase, raza, género como el resultado de largos procesos históricos, los cuales hay que ubicarlos en el campo de "conflictos sociales" y luchas por el poder. Actualmente, para las pedagogías críticas, estos conflictos hegemónicos son determinantes en la condición de la ciudadanía, en la formación moral, ya que la simple igualdad formal puede ser también otro mecanismo de discriminación social, racial o sexual.

En el campo de la educación multicultural, estas distintas posiciones se expresan en enfoques o tipologías que marcan ciertos acentos en la finalidad de la educación multicultural y en las formas de concebir la diversidad en el horizonte de la educación. Según las investigaciones de Gibson, Banks y Grant, en las sociedades hispanohablantes se pueden detectar por lo menos seis tipos de divergentes enfoques: 
a) Educar para igualar oportunidades: asimilación cultural. Se pretende igualar las "oportunidades educativas" para alumnos culturalmente diferentes y los presupuestos clave para postular esta visión son: 1) los niños culturalmente diferentes a la mayoría presentan dificultades de aprendizaje en instituciones educativas dominadas por esos valores culturales dominantes; 2) para remediar esas dificultades debe aumentar la compatibilidad entre escuela y hogar; 3) la asimilación cultural garantizará el éxito académico.

b) El entendimiento cultural a partir del conocimiento de la diferencia. Este enfoque apuesta por una educación que posibilite el conocimiento de las diferencias y no una educación de los denominados culturalmente diferentes. Es conveniente enseñar a todos a valorar y relevar la riqueza de las diferencias, sea de la cultura corriente dominante, de minorías u otras culturas. La escuela es un espacio que debe orientarse principalmente al enriquecimiento cultural de la comunidad académica. Este florecimiento se va logrando gracias a la visibilidad $y$ al conocimiento de la diversidad cultural. En el aula hay que asumir y aprender tanto las similitudes como las diferencias entre culturas, para preparar a los estudiantes a vivir en una sociedad multiétnica y multicultural.

c) El pluralismo cultural: preservary extender el pluralismo. Como rechazo a los procesos de "asimilación" cultural por parte de grupos sometidos a contacto con culturas mayoritarias, esta perspectiva insiste en la función de la educación multicultural en la "preservación" y la "extensión" del pluralismo cultural. Ni el "separatismo" ni la "asimilación" deben ser las finalidades de la educación. Se necesita un modelo de transmisión y diálogo cultural que fomente la amplitud, la profundidad y los intercambios entre los grupos culturales. Para que pueda extenderse el pluralismo se necesitan cuatro condiciones: 1) existencia de diversidad en la sociedad real; 2) interacción permanente intergrupos e intragrupos culturales; 3) coexistencia en condiciones de cierta equidad; 4) la sociedad en su conjunto debe convertir la valoración de la diversidad en un principio fundamental.

d) Educación bicultural o competencia por lo menos en dos culturas. La educación multicultural debe construir sujetos educativos competentes en dos culturas diferentes, ya sean éstas la "cultura nativa" y la "cultura dominante corriente", como también otras posibilidades. Las culturas no son necesariamente excluyentes, $y$ existen posibilidades on las que dos culturas se potencializan mutuamente, una especie de inter-bi-culturalidad. Para Spincer y Anda, la socialización bicultural adecuada exige ciertas condiciones como el papel de la lengua, destrezas en solución de problemas grupales, solapamientos culturales, posibilidades de "puentes", entre otras.

e) Educación multicultural como reconstrucción y transformación social. Se concibe el papel del multiculturalismo educativo como un proceso encaminado a elevar el nivel de conciencia de la comunidad académica en cuanto a su realidad social; a fortalecer una compresión crítica de la realidad. Especialmente, a crear mecanismos de "resistencia" y oposición de las culturas de los grupos no hegemónicos o contrahegemónicos. La educación ligada a la diversidad cultural tiene una función básica en el cambio social. Las instituciones y los mecanismos de control social y cultural (racismo, aporofobia, sexismo, clasismo, etc.), son criticados y sometidos a la resistencia de grupos sociales que plantean la transformación social emancipatoria. f) Educación anti-racista. La educación multicultural debe acentuar su función anti-racista en el proceso pedagógico; las diferencias culturales que no abordan el problema racial y de género terminan albergando la desigualdad. Para Alegret, una de las diferencias más importantes entre una educación multicultural no racista y una educación radicalmente anti-racista se encuentra en la forma de asumir las diferencias y desigualdades: para los no racistas las explicaciones de esta transformación son sólo "ignorancia y prejuicios", mientras para los anti-racistas esas transformaciones son problemas ideológicos que van mucho más allá de simples "patologías" o "prejuicios". La educación antiracista implica una estrategia muy sólida de intervención educativa que impida la "reproducción" cotidiana del racismo.

En un trabajo reciente del pedagogo español Xavier Besalú (2002), partiendo de la premisa de Eduardo Galeano de que "nunca han faltado pensadores capaces de elevar a categoría científica los prejuicios de la clase dominante", se llama la atención sobre la necesidad urgente de realizar un trabajo sistemático sobre el lenguaje, las actitudes y los valores del magisterio para combatir la discriminación en el aula de clase; y también se postula una tipología y currículum de educación intercultural. Para Besalú existen en términos generales tres perspectivas teórico-prácticas para abordar la diversidad cultural en la escuela: el asimilacionismo, el multiculturalismo y el interculturalismo.

El asimilacionismo parte de dos presupuestos en la orientación del trabajo educativo: dar prioridad a la unificación o cohesión social como finalidades para "superar" la fragmentación en las sociedades multiculturales, y considerar que la cultura escolar vigente es la cultura universal y válida para todos. Por tanto deben borrarse las diferencias y 
todos deben aprender lo mismo. Desde esta perspectiva teórica-práctica, la diversidad cultural se considera un problema por resolver y hasta un obstáculo. Tanto los alumnos minoritarios como los autóctonos pueden ver amenazados su nivel académico por la heterogeneidad cultural. Según esta visión, para evitar la distancia cultural y la deficiente adaptación, es necesario crear lo que ellos denominan programas compensatorios y aulas especiales.

El punto de partida y la finalidad central del multiculturalismo es el reconocimiento y la valoración de todas las culturas. Todas las culturas son diferentes e irreductibles. La pertenencia consciente a un grupo cultural aporta al individuo elementos positivos para su vida y formación educativa. También la coexistencia de grupos culturalmente diferenciados es considerada deseable, y propicia espacios de justicia social Las ópticas multiculturalistas son variadas y pueden ir desde la reivindicación de escuelas específicas para cada grupo o la convivencia de grupos diferenciados (esa convivencia también puede tener modelos y acentos).

La posición interculturalista plantea dos objetivos básicos: el pluralismo cultural concebido como el respeto por la identidad de cada cultura, y la construcción de una sociedad plural, cohesionada y democrática. Reconoce que la coexistencia de grupos culturales distintos es inevitablemente conflictiva, ya sea vía e antagonismo o la simple complementariedad. Propone evitar el aislamiento cultural y la fragmentación en que caen perspectivas multiculturalistas. Preservar la diversidad cultural significa que la escuela debe defender una cultura plural, en la que estén representadas todas las culturas que existen en un contexto determinado. La mayores dificultades de este enfoque están en si las relaciones son de "igualdad", si se posibilita la relativización y descentramiento de la propia cultura. Preservar la escuela intercultural implica enseñar a los alumnos a convivir en un ambiente culturalmente diverso y socialmente complejo y trabajar para construir un medio configurado con las aportaciones de todos.

Esperamos que este recorrido incremente nuestra conciencia sobre los complejos desafíos y las incertidumbres de una educación respetuosa del reconocimiento de la diferencia.

\section{BIBLIOGRAFÍA}

Bernstein, B. La construcción social del discurso pedagógico. Bogotá: Prodic, 1993.

Besalú, X. Diversidad cultural y educación. Madrid: Síntesis Educación, 2002.

Castells, M., Freire, P., Giroux, H. y OTROS. Nuevas perspectivas críticas en educación. Barcelona: Paidós Educador, 1994.

Colom, A. y Mélıch, J. Después de la modernidad: nuevas filosofías de la educación. Barcelona: Paidós, 1994.

Connell, R. W. Escuelas y justicia social. Madrid: Ediciones Morata, 1997.

DeloRs, J. La educación: encierra un tesoro. Madrid: Ediciones Unesco y Santillana, 1996.
Fraser, N. Justicia interrupta: reflexiones críticas desde la posición postsocialista. Bogotá: Siglo del Hombre y Universidad de los Andes, 1997.

García Castaño, J. y Granados, A. Lecturas para educación intercultural. Madrid: Trotta, 1999.

Gıroux, H. Placeres inquietantes: aprendiendo la cultura popular. Barcelona: Paidós Educador, 1996.

Gómez Buendía, H. (Dır.). Educación, la agenda del siglo XXI. Bogotá: Tercer Mundo Editores, 1998.

HALLAK, J. Invertir en el futuro. España: Ediciones Unesco y Tecnos, 1991.

Jordán, J. A. La escuela multicultural: un reto al profesorado. España: Paidós, 1994.
Kant, I. Tratado de Pedagogía. Bogotá: Ediciones Rosaristas, 1985.

McLaren, P. Pedagogía crítica, resistencia cultural y producción de deseo. Buenos Aires: Aique Grupo Editor, 1994.

Pedagogía, identidad y poder: los educadores frente al multiculturalismo. Buenos Aires: HomoSapiens, 1998.

Puiggrós, A. Imaginación y crisis en la educación latinoamericana. Buenos Aires: Aique Grupo Editor, 1994.

Torres S., JuRJo (comp.). Volver a pensar la educación. Madrid: Morata, 1995. 